\title{
IT-Service-Management - Ein Modell zur Bestimmung der Folgen von Interoperationalitätsstandards auf die Einbindung externer IT-Dienstleister
}

\author{
Kathrin Susanne Braunwarth
}

Bernd Heinrich

\section{Kernpunkte:}

Wie wirken sich Interoperationalisierungsstandards (IOS) wie Web-Service-Standards oder semantische Auszeichnungen auf die Auslagerung von Prozessen bzw. Prozessaktivitäten und damit die Einbindung externer IT-Dienstleister und deren Services aus? Dazu wird unter Berücksichtigung von Auszahlungs- und Risikoaspekten ein Entscheidungsmodell entwickelt, um Portfolios an (eigenerstellten oder fremdbezogenen) Services bilden zu können. Zentrale Ergebnisse auf Basis der definierten Annahmen sind:

- Lassen sich zukünftig durch IOS die Einbindungs- und Koordinationskosten für Services und Dienstleister reduzieren, so werden Unternehmen in ihrem Portfolio an IT-Services mehr Dienstleister einbinden, um die Risiken eines Service-Ausfalls zu senken.

- Können zudem auch die Umdisponierungskosten für den Ersatz ausgefallener Services reduziert werden, so entsteht bei Service-Ausfällen geringerer ökonomischer Schaden. In diesem Fall verliert die Bildung von Portfolios an IT-Services mit mehreren Services unter Risikogesichtspunkten an Bedeutung und es genügt im Extremfall, jeweils den Günstigsten anzufordern.

- Durch das heute oftmals übliche, vollständige Auslagern eines Prozesses an einen Dienstleister wird im Sinne des hier vorgestellten Ansatzes Optimierungspotenzial verschenkt. Besteht die Möglichkeit einzelne Aktivitäten bzw. sogar einzelne Aktivitätsdurchführungen unabhängig voneinander zu vergeben, dann lässt sich damit das Ausfallrisiko diversifizieren.

\section{Stichworte:}

Web Services, Ausfallrisiken, Abhängigkeiten, IT-Dienstleister, Sourcing

\section{Zusammenfassung:}

Im Beitrag wird untersucht, wie sich Interoperationalitätsstandards wie Web-ServiceStandards oder semantische Auszeichnungen von Services auf die Auslagerung von Prozessen bzw. Prozessaktivitäten und damit die Einbindung externer IT-Dienstleister und deren Services auswirken. Dazu wird ein Entscheidungsmodell entwickelt, um unter Berücksichtigung von Auszahlungs- und Risikoaspekten (bspw. Ausfälle fremdbezogener Services) ein ServicePortfolio ermitteln zu können. Illustriert wird das Modell beispielhaft anhand eines Ausschnitts des Prozesses zur Girokontoeröffnung. 


\title{
IT-Service-Management - A Model for determining the Impact of Interoperability Standards on the Integration of external IT Service Providers
}

\begin{abstract}
:
This article examines the impact of interoperability standards such as Web Service standards or semantic annotation of services on the outsourcing of business processes or process activities. Especially the integration of external IT service providers is considered. Therefore a decision model is developed for optimizing service portfolios regarding risk-/cost-aspects. By using an extract of the application process for current accounts we exemplify the implementation and the results of the presented model.
\end{abstract}

Keywords: Web Services, supply uncertainty, dependence, IT Service Provider, Sourcing 


\section{Einleitung}

Der führende Anbieter von On-Demand-CRM-Diensten Salesforce stellt seinen Kunden eine Online-Vertriebsplattform für Business-Applikationen zur Verfügung. Als es jedoch Ende 2005 innerhalb von sechs Wochen zu drei massiven Systemausfällen bei Salesforce kam (Buck 2006, S. 11), standen die Kunden vor ernsthaften Problemen. Sie konnten ihre eigenen Prozesse nicht oder nur eingeschränkt durchführen. Einzelne Kunden konnten zwar auf einen alternativen Dienstleister ausweichen, dabei fielen jedoch hohe Kosten für den Wechsel an.

Wie in diesem Beispiel sind heute viele Unternehmen infolge der starken Vernetzung vital von den Dienstleistungen ihrer Lieferanten abhängig (vgl. auch die Fälle bei Ericsson und Nokia; Latour 2001). Verschärft wird diese Situation durch die zunehmende Bedeutung des Business Process Outsourcing (BPO): Hier prognostiziert Forrester Research für die nächsten fünf Jahre in Europa eine durchschnittliche Zuwachsrate von 11,5\% und damit die höchste aller betrachteten IT-Service Bereiche. Demnach wird der Markt von 11 Milliarden $€$ in 2006 bis 2011 auf 18,9 Milliarden $€$ wachsen (Takahashi et al. 2006). Auch kritische Prozesse werden als Ganzes oder weitgehend an einzelne Dienstleister vergeben. Unternehmensinternes Know-how über den Prozessablauf geht dadurch oftmals verloren (Reinicke 2005, S. 130f) und die Umsetzung neuer Anforderungen wird - da ein Dienstleister diese häufig nicht versteht bzw. verstehen kann - erschwert. Der Bezug von IT-Dienstleistungen aus nur einer Quelle schafft ferner Abhängigkeiten, die Risiken und hohe (Wechsel-)Kosten implizieren können.

Um zur Lösung derartiger Probleme beizutragen, wird die schnelle und flexible Einbindung von IT-Dienstleistungen in die eigenen Prozesse (bspw. Stichwort „on-demand Computing“) intensiv diskutiert (Reinicke 2005, S. 129ff). Auch Service-Märkte wie sie von (Eymann, Neumann et al. 2006, S. 5) postuliert werden, versprechen hierzu Unterstützung. Vor diesem Hintergrund beschäftigt sich der Beitrag mit der Frage, wie sich IOS (bspw. Web-ServiceStandards) auf die Abhängigkeit von Lieferanten auswirken und wie insbesondere die damit verbundenen Risiken im Rahmen eines IT-Service-Managements besser gesteuert werden können.

Der Beitrag ist wie folgt aufgebaut: Kapitel 2 beschreibt die Abhängigkeiten, die bei der Einbindung externer IT-Dienstleister entstehen und diskutiert, wie sich Interoperationalitätsstandards (IOS) zukünftig auf diese Abhängigkeiten auswirken könnten. Danach wird ein Überblick über bisherige Arbeiten gegeben. Die Abhängigkeiten und die damit verbundenen Risiken sind zugleich Ausgangspunkt, um in Kapitel 3 ein Entscheidungsmodell zur Optimierung der IT-Service-Auswahl unter Kosten-/Risikoaspekten zu entwickeln. Die Modellanwendung wird in einem Fallbeispiel in Kapitel 4 verdeutlicht. Das Kapitel 5 fasst die Implikationen aus dem Modell zusammen und würdigt diese kritisch. 


\section{Prozessdurchführung mit IT-Services}

Durch eine umfangreiche Einbindung externer IT-Dienstleister in die Unternehmensprozesse bzw. durch Auslagerung gesamter Prozesse steigt die Abhängigkeit von Dienstleistern. Was bedeutet hier jedoch konkret Abhängigkeit? Welche Abhängigkeiten in Bezug auf die Durchführung von Prozessen zu unterscheiden sind, wird in Abschnitt 2.1 diskutiert, während Abschnitt 2.2 erläutert, wie diese - gerade vor dem Hintergrund neuer IOS - verringert werden können. In Abschnitt 2.3 wird hierzu bestehende Literatur aufgearbeitet.

\subsection{Analyse der Abhängigkeiten von Dienstleistern}

Die Abhängigkeit von externen IT-Dienstleistern lässt sich im Wesentlichen in zwei Gruppen unterscheiden. Zum einen kann es nach Auslagerung eines Prozesses beim Bezug der Leistung zu Problemen kommen, d. h. der IT-Lieferant kann seine Dienstleistung nicht oder nicht in der vereinbarten Qualität, Zeit oder Menge bereitstellen. Die Gründe hierfür können vielfältig sein und reichen von Ursachen, die vom Dienstleister nicht zu vertreten sind (bspw. Naturkatastrophen, Terroranschläge, (vgl. Kleindorfer und Saad 2005, S. 1f; Tomlin 2006, S. 639f) bis hin zu organisatorischen oder technischen Unzulänglichkeiten des Dienstleisters. Da bei einem (unerwarteten) Ausfall in der Regel nicht flexibel Dienstleistungen anderer Lieferanten eingebunden werden können, besteht das Risiko, dass Teile des Geschäftsbetriebs nicht aufrechterhalten werden können. Zum Beispiel kann ein Finanzdienstleister (FDL) nicht über die Vergabe eines Kredits entscheiden, ohne dass die Dienstleistung ,, Rating des Kunden “ zur Verfügung steht.

Die zweite Gruppe von Abhängigkeiten resultiert nicht aus dem möglichen Ausfall einer Dienstleistung, sondern betrifft die mangelnde Flexibilität des Lieferanten, falls sich Anforderungen an die bereit zu stellenden Services ändern, oder die fehlende Flexibilität beim Lieferantenwechsel selbst. Aus dieser Art der Abhängigkeit kann folgen, dass bspw. Produktinnovationen, die zu geänderten Anforderungen führen, nicht, nur verspätet oder nur mit hohen Kosten realisiert werden können. Entwickelt bspw. ein FDL ein Kreditprodukt, das online abschließbar sein soll, wofür aber eine weitergehende Bonitätsbeurteilung des Kunden nötig ist, so kann dieses nur eingeführt werden, falls der externe Anbieter eines Ratingservice diese Informationen auch zur Verfügung stellt.

Für beide Gruppen von Abhängigkeiten lassen sich in der Literatur eine Reihe von Beispielen finden. Ein Beispiel für die erste Gruppe ist der beschriebene Fall des Mietsoftwareanbieters Salesforce (Buck 2006, S. 11). Fragt man hier nach der optimalen Auswahl von IT-Services verschiedener Lieferanten, so sind nicht nur die Kosten für den Service, sondern auch die Wahrscheinlichkeiten für deren Ausfall als Risiko zu berücksichtigen. Jedoch ist die Abhängigkeit nicht nur auf die Ausfallwahrscheinlichkeit für einzelne Services zu reduzieren. Gerade beim Eintritt größerer Zwischenfälle (bspw. vollständige Systemausfälle) besteht die Gefahr, dass nicht nur ein Service davon betroffen ist, sondern mehrere Services, ggf. auch unterschiedlicher Lieferanten. Wenn demnach kritische Prozesse an einen einzelnen Dienstleister oder miteinander verbundene ausgelagert werden, entstehen so genannte 
„Klumpenrisiken“, d. h. vom Ausfall eines Lieferanten ist nicht nur ein Service, sondern mehrere dieses oder „,verbundener“ Lieferanten betroffen. Gerade im IT-Bereich resultieren derartige Risiken jedoch nicht nur aus größeren Katastrophen, sondern auch aus der Größe und Komplexität der technischen Infrastruktur und deren Zuverlässigkeit und Sicherheit.

Die zweite Gruppe von Abhängigkeiten wirkt sich wie folgt aus: Die Vergabe von Prozessen an einen externen Dienstleister ist infolge der starken Vernetzung der Prozesse und ITSysteme im eigenen Unternehmen oftmals eine kosten- und zeitintensive Aufgabe. Hier müssen neben der Anpassung von Systemen auch bestehende Schnittstellen modifiziert bzw. neue Schnittstellen definiert werden. Zwar rechnet sich oftmals die initiale Vergabe noch, spätestens wenn der einmal eingebundene Dienstleister wieder gewechselt werden soll, wird die Abhängigkeit deutlich. Ein Wechsel und damit eine erneute Anpassung der eigenen Strukturen sind unter wirtschaftlichen Gesichtspunkten oftmals nicht zu rechtfertigen. Auf diese Weise entsteht eine nicht zu unterschätzende Abhängigkeit, wenn hohe Folgekosten für einen Wechsel vermieden werden sollen. Ein weiterer wichtiger Punkt ist, dass mit der Vergabe von Prozessen häufig ein Verlust an Wissen einhergeht (Reinecke 2005, 129ff), der die eigene Gestaltungskompetenz für die Prozessanpassungen und -änderungen verringert. Letzteres erhöht die Abhängigkeit von externen Dienstleistern, da selbst bei einem beabsichtigten Wechsel das Unternehmen teilweise gar nicht mehr die Kompetenz zur Durchführung der „eigenen“ Prozesse besitzt.

Daneben können auch viele kleinere Änderungsanforderungen zu Problemen führen. Bspw. begründete die Verkehrsbank ihren Ausstieg aus einem BPO-Kontrakt mit der mangelnden Reaktionsfähigkeit des Lieferanten auf eine veränderte Geschäftstätigkeit (Racky 2005). Ähnlich erging es dem Schuhhersteller Salamander, der Ende der 90er Jahre seine ITDienstleistungen vollständig von einem Lieferanten bezog. Allerdings sah der Zehn-JahresVertrag keine Anpassung an wirtschaftliche Rahmenbedingungen wie bspw. neue Kapazitäten, Mindestmengen oder Auslastungen vor (o. V. 2005). Die dabei bemängelte Inflexibilität begründet sich entweder mit der Unfähigkeit des Lieferanten auf Anforderungen schnell und zielgerichtet zu reagieren, oder aber diese mit vertretbarem Aufwand umzusetzen. Zusammenfassend zielt die zweite Gruppe von Abhängigkeiten damit auf die (hohen) Wechsel- und Einbindungskosten ab.

\subsection{Einfluss von Interoperationalitätsstandards}

Wie eingangs beschrieben, ist der Einfluss von IOS auf die dargestellten Abhängigkeiten zu diskutieren. Die Grundidee ist dabei, einen Prozess oder mehrere nicht mehr komplett an einen Dienstleister zu vergeben. Vielmehr sind die Prozesse in einzelne Aktivitäten (granular) zu untergliedern, über deren Vergabe dann entschieden werden kann - sofern strategische Überlegungen oder technische Gegebenheiten nicht dagegen sprechen. Eine einzelne Aktivität lässt sich z. B. durch einen Service im technologischen Sinne, wie bspw. einen Web-Service realisieren. Unter Service wird somit ein softwaretechnisch realisiertes Artefakt zum Anbieten einer Funktionalität verstanden. So kann ein Service die Funktionalität zur Durchführung einer IT-gestützten Prozessaktivität oder mehrerer kapseln. Insofern wird hier auch von Modul- 
arisierung der Prozesse gesprochen, da die Services zur Realisierung von Aktivitäten schneller und kostengünstiger ausgetauscht oder fremdbezogen werden sollen. Ein weiterer Vorteil dieser Services kann darin liegen, dass sie sich an definierten Standards orientieren. Der Beitrag fokussiert daher an sich standardisierte und austauschbare Services, die bspw. eine Schufa-Abfrage oder Online-Bezahldienste (z. B. paypal oder giropay) kapseln. Selbst diese Services werden sich jedoch realistischerweise bspw. in ihrer Verfügbarkeit oder Lieferzuverlässigkeit unterscheiden (in einigen Bereichen versuchen Lieferanten sich gerade hier zu differenzieren). Aspekte wie Verfügbarkeit oder Lieferzuverlässigkeit können dann wiederum als Risiko verstanden werden, d. h. es besteht eine Abhängigkeit von den IT-Dienstleistern. Und selbst wenn sich die Preise der Services angleichen würden, wäre gerade die Berücksichtigung des Risikos in Form einer eingeschränkten Zuverlässigkeit und damit der Risikodiversifikation interessant, da es bei einer Einbindung mehrerer IT-Dienstleister zu Abwägungen beispielsweise zwischen einem geringeren Risiko des Ausfalls und der geringeren Abhängigkeit bei mehreren Dienstleistern und den dafür ggf. insgesamt höheren Einbindungskosten kommt. Welchen Einfluss IOS hierbei haben können, wird im Folgenden beschrieben.

Auszeichnungsstandards für Services: Monolithische, stark integrierte IT-Systeme bzw. die proprietäre Vernetzung von Systemen führen dazu, dass oftmals nur mit hohem Aufwand eine Einbindung von Dienstleistern möglich ist. Wenn hierbei keine Standardisierung erfolgt, erhöht dies den zukünftigen Aufwand bei einem Anbieterwechsel oder bei Änderungsanforderungen. Mittels Aufbau, Kommunikation und externen Schnittstellen der Services sowie deren Auszeichnung nach definierten Standards wird versucht, dem entgegen zu wirken, auch wenn dadurch die Einbindungskosten natürlich nicht völlig vermieden werden können. Für eine (syntaktische) Beschreibung der Services kann bspw. auf Standards wie WSDL zurückgegriffen werden.

Service-Choreographie: Bedingt durch hohe Kosten bei der Einbindung von Dienstleistern werden heute oftmals gesamte Prozesse extern vergeben. Dies erfolgt zum einen mit dem Ziel, derartige „einmalige Investitionen“ durch die angestrebten Skaleneffekte zu amortisieren. Besteht zum anderen allerdings die Notwendigkeit, die Prozesse häufig an veränderte Umweltbedingungen schnell und effizient anzupassen, so kann dies ggf. flexibler durch die Kombination von Services verschiedener Lieferanten erreicht werden. Laut van der Aalst (2003, S. 74) und Bruijn, Fensel et al. (2005, S. 46) gilt dies insbesondere, falls sich bspw. die Ablaufreihenfolge oder die Funktionalität einer Aktivität ändern. Da eine Aktivität i. d. R. durch mehrere alternative Services realisiert werden kann, ergeben sich zusätzliche Möglichkeiten der Durchführung einer Aktivität bzw. eines Prozesses. Diese „Service-Choreographie“ (Aalst 2003, S. 74; Bruijn, Fensel et al. 2005, S. 46) kann dabei unternehmensintern erfolgen. Dies bedeutet, dass die Service-Ausführung unter Berücksichtigung von Datenintegrationsaspekten zwar unternehmensextern stattfinden kann, das Prozess-Know-how und die Gestaltungskompetenz aber im Unternehmen bleiben. Idealtypisch sollen so Inflexibilitäten in Form einer mangelnden Reaktionsfähigkeit durch Wechsel des Dienstleisters oder durch eine Kombination verschiedener Dienstleister reduziert werden können. Letzteres reduziert die Abhängigkeit von einem Anbieter. 
Service-orientierte Architekturen (SOA): Die Service-Choreographie zur IT-gestützten Prozessdurchführung kann ggf. in einem ersten Schritt die Flexibilität bspw. bei einer Anforderungsänderung steigern, jedoch geht bei einer Vielzahl genutzter Services der Überblick schnell verloren. Für ein effizientes Management der genutzten Services bedarf es deshalb neben den beschriebenen Auszeichnungsstandards für Services eines architektonischen Rahmens, der bspw. das Auszeichnen, Finden und Einbinden der Services regelt. Einen solchen Rahmen sollen SOA zukünftig bieten. Das Konzept der SOA sieht lose gekoppelte Services als Bestandteile vor, die ihre Dienste registrieren und zugleich über standardisierte Schnittstellen zur Verfügung stellen. Die Benutzung der Services wird dabei über einen Dienstmakler organisiert. Services können so in verschiedenen Prozessen mehrmalig verwendet werden. Ändert sich ein Service, so wird transparent, in welchen Prozessen dieser bisher genutzt wurde. Diese Eigenschaften von SOA sollen Unternehmen ein erhöhtes Maß an Flexibilität geben, um auf Umweltänderungen reagieren zu können. Zudem soll durch ein effizientes Management der Services und die Transparenzschaffung die Reaktionsfähigkeit erhöht werden.

Semantische Service-Auszeichnungen: Heutzutage erfolgt die Suche nach geeigneten Services, deren Einbindung und Management größtenteils manuell. Um diese Tätigkeiten zukünftig (teilweise) zu automatisieren und dadurch die Umdisponierungskosten bspw. bei einem (unerwarteten) Service-Ausfall reduzieren zu können, werden semantische ServiceAuszeichnungen (bspw. mittels OWL-S, vgl. Lara, Ruben et al. 2004, S. 254-269) diskutiert. Weiterhin soll durch den Einsatz von Ontologien und hier die Nutzung von Klassen und Beziehungen eine semantische Vergleichbarkeit erzielt werden. Dies könnte zukünftig zum einen zusammen mit Algorithmen der Semantic Web-Service-Komposition zu einer (Teil-)Automatisierung der Integration von Services zur Prozessdurchführung beitragen. Zum anderen soll auch eine schnelle Reaktion auf Service-Ausfälle ermöglicht werden, indem automatisiert ein Ersatzservice gesucht wird. Um den bestmöglichen Service auswählen zu können, ist es zudem notwendig, nichtfunktionale Größen wie z. B. Durchführungs- und Wartezeiten, Preis, Zuverlässigkeit und Qualität sowie etwaige Service-Levels zu hinterlegen (für Details vgl. Ran (2003, S. 1-10)). Letzteres weist bereits auf die Notwendigkeit von Vereinbarungen (Service Level Agreements etc.) hin, wie sie bspw. schon früh von Meyer (1990, S. 120ff) mit seinem Konzept des „Design by Contract“ bereits vorgeschlagen wurden. Hier sind die Beziehungen zwischen Komponenten als „formale Vereinbarungen [...], in der die Rechte und Pflichten jeder Partei festgelegt sind“ aufzufassen. Zur Realisierung dieser Ziele wird jedoch noch einiges an Entwicklungsaufwand - gerade im Bereich der semantischen Konzepte notwendig sein.

Um die beschriebenen Potenziale zukünftig zu realisieren, müssen Service-Konzepte im Hinblick auf Eigenschaften erweitert werden, wie sie auch schon teilweise bei verteilten Systemen diskutiert wurden. Hier sind Transparenz, Offenheit, Skalierbarkeit, Sicherheit, Konsistenz, Fehlertoleranz oder Datenintegrität zu nennen. Datenintegrität fordert in diesem Kontext, dass Services zum Beispiel auf einen gemeinsamen, konsistenten Datenbestand zugreifen oder aber diese durch zusätzliche Instrumente (wie z. B. einem Enterprise Service Bus) sichergestellt wird. Derartige Probleme sind zu lösen, bevor die IOS zukünftig ihren Lösungsbeitrag erbringen können (auch deshalb wurde oben von Potenzialen gesprochen). Nichtsdes- 
totrotz zeigen bereits heute einzelne prototypische Implementierungen bspw. im Rahmen des EU-Projekts SUPER, welche Vorteile sich dadurch ergeben können.

Wie wirken sich nunmehr die IOS - unter der Annahme ihrer Etablierung - künftig auf die in Abschnitt 2.1 beschriebenen Abhängigkeiten aus? Durch Standardisierung der ServiceAuszeichnungen kann besser über die Auslagerung einzelner Aktivitäten entschieden werden. Demzufolge ist es nicht mehr notwendig, Prozesse komplett zu vergeben. Im Extremfall kann für jede einzelne Prozessaktivität entschieden werden, ob sie weiterhin im Unternehmen durchgeführt - bspw. weil strategische Überlegungen wie Wettbewerbsrelevanz dagegen sprechen - oder von einem oder mehreren Lieferanten erbracht werden soll. Dies ist möglich, weil die Services unterschiedlicher Lieferanten infolge der Standards besser eingebunden werden können. Letzteres weist bereits daraufhin, dass nun auch eine effiziente Aufteilung der Nachfrage auf mehrere Lieferanten möglich ist. Während es heute häufig noch notwendig ist, bspw. verschiedene Schnittstellen(-technologien) einzuführen und zu pflegen, falls mehrere Lieferanten eingebunden werden sollen, könnte das künftig durch Standards reduziert wenn auch nicht gänzlich vermieden - werden. Mit Hilfe von Service-Choreographie und SOA lassen sich zudem Reaktionszeiten und Umdisponierungskosten bei der Suche nach Ersatz-Services im Fall eines Ausfalls reduzieren. Analog dazu können auch Einbindungskosten für einen neuen Dienstleister bzw. für einen neuen Service verringert werden. Basis hierfür ist, dass neue Dienstleister sich mit ihrem Leistungsangebot im Diensteverzeichnis registrieren lassen. Durch die hierbei erfolgte Offenlegung der Schnittstellen hat das Unternehmen bei Bedarf schneller Zugriff. Darüber hinaus könnte die semantische Service-Auszeichnung sowie darauf basierende Quality-of-Service-Vereinbarungen - wegen einer dann möglichen (teil-)automatisierten Einbindung von Services - noch zu einer weiteren Reduktion der Umdisponierungs- und Einbindungskosten führen. Bild 1 illustriert nochmals die Aspekte der diskutierten zukünftigen Entwicklungen.

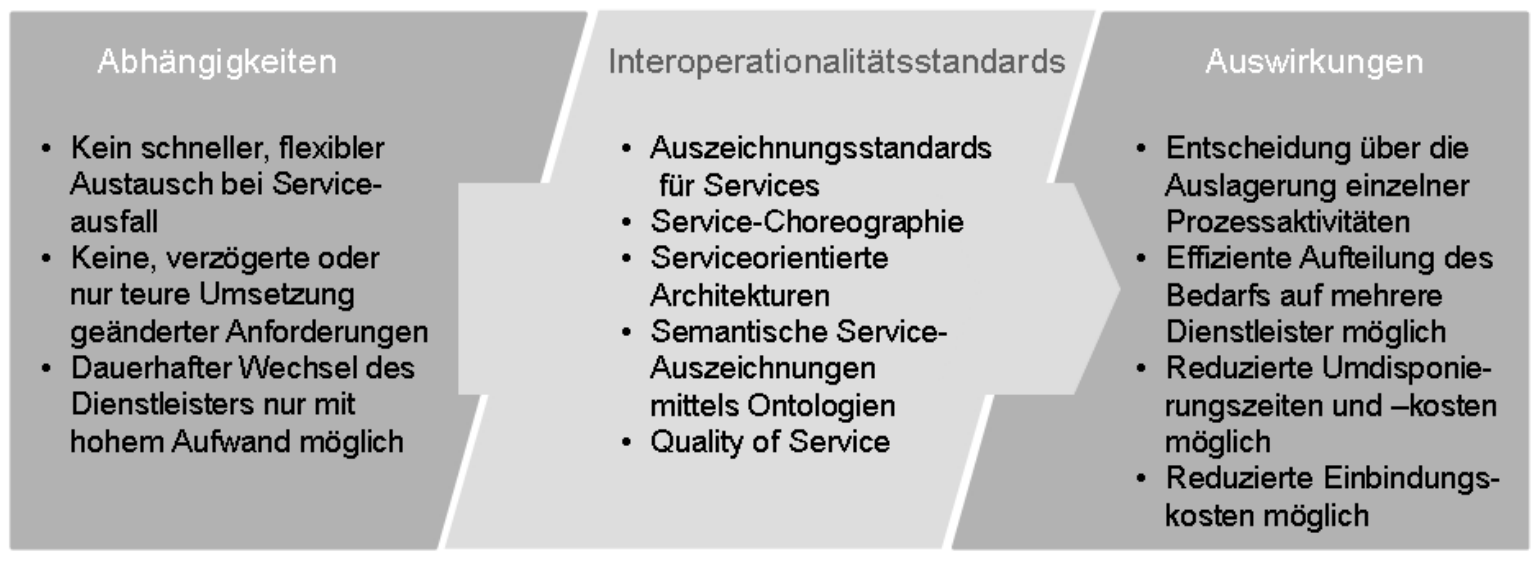

Bild 1: Auswirkungen von Interoperationalitätsstandards

\subsection{Bisherige Arbeiten}

Auf Basis der Zusammenhänge in Bild 1 werden die Arbeiten von Knolmayer (1991, S. 323341), Wirth (1996), Martínez-de-Albéniz und Simchi-Levi (2005, S. 90-114), Kleindorfer und 
Saad (2005, S. 53-68) und Tomlin (2006, S. 639-657) in den nachfolgenden Tabellen kurz gegenüber gestellt. So soll identifiziert werden, welcher Teilaspekt der Problemstellung bereits untersucht wurde und wo noch Forschungsbedarf besteht.

Tabelle 1: Gegenüberstellung bisheriger Arbeiten hinsichtlich der Abhängigkeiten

\begin{tabular}{|c|c|c|c|c|c|c|}
\hline & $\begin{array}{l}\text { Knolmayer } \\
\text { (1991) }\end{array}$ & Wirth (1996) & $\begin{array}{c}\text { Martinez-de- } \\
\text { Albéniz und } \\
\text { Simchi-Levi (2005) }\end{array}$ & $\begin{array}{l}\text { Kleindorfer und } \\
\text { Saad (2005) }\end{array}$ & Tomlin (2006) \\
\hline \multirow{3}{*}{ 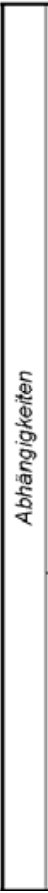 } & \multirow{2}{*}{ 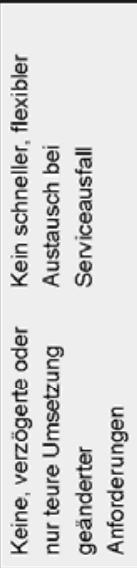 } & \multirow[t]{2}{*}{$\begin{array}{l}\text { Werden nicht } \\
\text { betrachtet. } \\
\text { Abhängigkeit } \\
\text { wird lediglich als } \\
\text { Schwellwert } \\
\text { repräsentiert }\end{array}$} & $\begin{array}{l}\text { Ausfallrisiken } \\
\text { werden zwar } \\
\text { qualitativ } \\
\text { differenziert, im } \\
\text { Modell jedoch von } \\
\text { einer Variablen } \\
\text { repräsentiert. }\end{array}$ & $\begin{array}{l}\text { Ausfallrisiken } \\
\text { werden nicht } \\
\text { betrachtet. Es } \\
\text { geht darum, das } \\
\text { richtige Volumen } \\
\text { für unbekannten } \\
\text { Bedarf zu } \\
\text { bestellen. }\end{array}$ & $\begin{array}{l}\text { Ausfallisisiken } \\
\text { werden als } \\
\text { zunehmend } \\
\text { zentrales Problem } \\
\text { für Wertschöpf- } \\
\text { ungsketten } \\
\text { identifiziert. } \\
\text { Formulierung von } \\
\text { Prinzip } 3\end{array}$ & $\begin{array}{l}\text { Wird durch eine } \\
\text { sog. Volumen- } \\
\text { Flexibilitäts- } \\
\text { funktion } \\
\text { abgebildet. }\end{array}$ \\
\hline & & & Nicht modelliert & $\begin{array}{l}\text { Es werden } \\
\text { lediglich } \\
\text { hinsichtlich des } \\
\text { Volumens des } \\
\text { Inputprodukts } \\
\text { geänderte } \\
\text { Anforderungen } \\
\text { betrachtet. }\end{array}$ & Nicht betrachtet & Nicht modelliert \\
\hline & 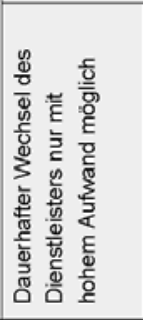 & $\begin{array}{l}\text { Nur } \\
\text { Umstellkosten } \\
\text { zwischen } \\
\text { Eigenerstellung } \\
\text { und } \\
\text { Fremdbezug } \\
\text { modelliert; nicht } \\
\text { Wechsel } \\
\text { zwischen } \\
\text { Dienstleistern. }\end{array}$ & Nicht betrachtet & Nicht betrachtet & Nicht betrachtet & Nicht betrachtet \\
\hline
\end{tabular}


Tabelle 2: Gegenüberstellung bisheriger Arbeiten hinsichtlich der Auswirkungen

\begin{tabular}{|c|c|c|c|c|c|c|}
\hline & $\begin{array}{c}\text { Knolmayer } \\
\text { (1991) }\end{array}$ & Wirth (1996) & $\begin{array}{c}\text { Martinez-de- } \\
\text { Albeniz und } \\
\text { Simchi-Levi } \\
(2005)\end{array}$ & $\begin{array}{c}\text { Kleindorfer } \\
\text { und Saad } \\
(2005)\end{array}$ & Tomlin (2006) \\
\hline \multirow{4}{*}{ 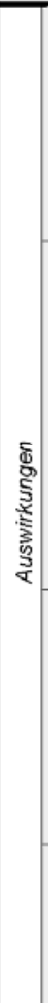 } & 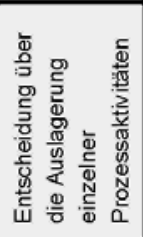 & $\begin{array}{l}\text { Entscheidungen } \\
\text { über Eigen- } \\
\text { erstellung oder } \\
\text { Fremdbezug von } \\
\text { elementaren } \\
\text { IT-Aufgaben. }\end{array}$ & $\begin{array}{l}\text { Keine Angaben } \\
\text { zur Granularität } \\
\text { der } \\
\text { auszulagernden } \\
\text { Einheiten }\end{array}$ & $\begin{array}{l}\text { Keine Angaben } \\
\text { zur } \\
\text { Granularität. } \\
\text { Es wird ganz } \\
\text { allgemein ein } \\
\text { Inputprodukt } \\
\text { betrachtet. }\end{array}$ & $\begin{array}{l}\text { Formulierung } \\
\text { von Prinzip } 9\end{array}$ & $\begin{array}{l}\text { Keine Angaben } \\
\text { zur Granularität. } \\
\text { Es werden ganz } \\
\text { allgemein } \\
\text { zugelieferte } \\
\text { Produkte } \\
\text { betrachtet. }\end{array}$ \\
\hline & 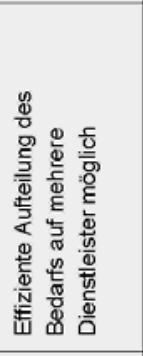 & $\begin{array}{l}\text { Nicht möglich. Es } \\
\text { wird binär } \\
\text { zwischen } \\
\text { Eigenerstellung } \\
\text { und Fremdbezug } \\
\text { einer jeden } \\
\text { Leistung } \\
\text { entschieden. }\end{array}$ & $\begin{array}{l}\text { Nicht möglich. } \\
\text { Lediglich } \\
\text { beliebige } \\
\text { Anteilsverteilung } \\
\text { zwischen } \\
\text { Eigenerstellung } \\
\text { und Fremdbezug. }\end{array}$ & $\begin{array}{l}\text { Portfoliobildung } \\
\text { mehrerer } \\
\text { Verträge/ } \\
\text { Beschaffungs- } \\
\text { arten, im Sinne } \\
\text { einer Gewinn- } \\
\text { maximierung } \\
\text { zu optimieren; } \\
\text { nicht aber } \\
\text { Aufteilung auf } \\
\text { Dienstleister. }\end{array}$ & $\begin{array}{l}\text { Formulierung } \\
\text { von Prinzip } 2 .\end{array}$ & $\begin{array}{l}\text { Beliebige } \\
\text { Anteilsverteilung } \\
\text { zwischen } \\
\text { mehreren } \\
\text { Lieferanten. Im } \\
\text { Beitrag wird } \\
\text { exemplarisch } \\
\text { von zwei } \\
\text { Lieferanten } \\
\text { ausgegangen. }\end{array}$ \\
\hline & 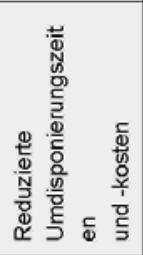 & $\begin{array}{l}\text { Anteilige } \\
\text { Umstellungs- } \\
\text { kosten können in } \\
\text { Koeffizienten für } \\
\text { die Kosten einer } \\
\text { Bereitstellungs- } \\
\text { form einbezogen } \\
\text { werden. }\end{array}$ & Nicht modelliert & Nicht modelliert & $\begin{array}{l}\text { Nicht } \\
\text { betrachtet }\end{array}$ & $\begin{array}{l}\text { Annahme } \\
\text { schließt Kosten } \\
\text { für Umdispo- } \\
\text { nierung aus. }\end{array}$ \\
\hline & 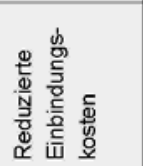 & Nicht modelliert & Nicht modelliert & Nicht modelliert & $\begin{array}{l}\text { Nicht } \\
\text { betrachtet }\end{array}$ & Nicht modelliert \\
\hline
\end{tabular}

Wie die Tabellen verdeutlichen, existiert zum einen eine noch zu schließende Lücke hinsichtlich der Betrachtung der Abhängigkeiten. Keiner der Ansätze unterscheidet hier zwischen Ausfall, verzögerter Umsetzung veränderter Anforderungen und Lieferantenwechsel. Auch bzgl. der Aufteilung auf mehrere Lieferanten lässt sich Folgendes feststellen: Während Knolmayer (1991) noch eine binäre Entscheidung zwischen Eigenerstellung und Fremdbezug modelliert, ist bei Wirth (1996) bereits die anteilsmäßige Verteilung auf die beiden Alternativen Eigenerstellung und Fremdbezug möglich. Der Portfolioansatz von Martínez-de-Albéniz und Simchi-Levi (2005) erlaubt die Verteilung auf verschiedene Vertragstypen, betrachtet aber nicht mehrere Leistungen. Im Modell von Tomlin (2006) ist dies zwar möglich, allerdings geht er nicht auf eine Portfoliobildung ein, sondern betrachtet einzelne Produkte und deren mengenmäßige Aufteilung auf verschiedene Lieferanten isoliert. Kleindorfer und Saad (2005) widmen sich v. a. Prinzipien zur Reduzierung des Risikos eines Leistungsausfalls. Keine der Arbeiten kommt jedoch hinsichtlich der Fragestellung, in welchem Umfang die benötigten Services bei verschiedenen Lieferanten bezogen werden sollen, zu einem Optimum hinsichtlich Auszahlungen und Risiko (insbesondere Ausfallrisiko).

\section{Formulierung des Entscheidungsmodells}

Im Folgenden wird ein Entscheidungsmodell zur Bestimmung des optimalen IT-ServicePortfolios (ITSP) entwickelt. Als Grundlage dienen Dokumentationen von Prozessen, die durch Services grundsätzlich realisierbar sind. Anhand dieser Dokumentationen wird ermit- 
telt, wie häufig jede Aktivität innerhalb der Betrachtungsperiode ausgeführt wird (vgl. hierzu bspw. Blodig et al. (2006, S. 473-486)) sowie welche Aktivitäten manuell bzw. durch (fremdbezogene oder eigenerstellte) Services auszuführen sind. Dabei sind strategische Überlegungen und Entscheidungen zum Outsourcing (Wettbewerbsrelevanz etc.) zum Optimierungszeitpunkt bereits abgeschlossen, d. h. es steht fest, ob bestimmte Prozesse bzw. einzelne Aktivitäten grundsätzlich durch extern bezogene Services durchgeführt werden „dürfen“. Das Modell greift auf portfoliotheoretische Überlegungen von Markowitz (1959) zurück und erweitert diese, um ein ITSP zu generieren, das die Auszahlungen und Risiken der Services sowie zugehörigen Mengen berücksichtigt. Folgende Annahmen werden hierzu getroffen:

(A.1) Die Häufigkeit $i_{m} \in\{0,1,2, \ldots\}$ der Durchführung einer jeden Aktivität $m \in\{1 \ldots M\}$ in der Betrachtungsperiode ist gegeben. Jede Aktivität $m$ lässt sich durch den Service $a_{m, n}$ eines Lieferanten $n \in\{1,2, \ldots, N\}$ realisieren. Ein Element der $M x N$-Matrix $A$ (Angebotsmatrix) mit $a_{m, n} \in\{0 ; 1\}$ ist mit eins belegt, falls der Lieferant $n$ für Aktivität $m$ einen Service anbietet, anderenfalls mit null. Die Entscheidungsvariablen des Modells sind die Anteile $x_{m, n} \in[0 ; 1]$ im ITSP, d. h. wie oft ein Service $a_{m, n}$ bezogen auf alle Ausführungen $i_{m}$ einer Aktivität $m$ eingebunden werden soll.

Da für die Einbindung und Nutzung eines Service Kosten anfallen, wird angenommen:

(A.2) Für eine Einheit des Service $a_{m, n}$ ist der Preis $p_{m, n}$ (mit $p_{m, n} \in R$ ) zu entrichten. Daneben sind Einbindungskosten $C_{a_{\mathbf{v}, n}}$ für einen Service des Lieferanten $n$ zu berücksichtigen. Diese errechnen sich mittels des Kostensatzes $c_{a_{\mathbf{v}, n}}$ für die erstmalige Einbindung jedes Services des Lieferanten $n$. Aufgrund von Erfahrungswerten - im Modell repräsentiert durch den Exponenten $\gamma \in] 0 ; 1]$ - sollen die Grenzkosten der Einbindung für jeden weiteren Service des Lieferanten $n$ (realistischerweise) kontinuierlich fallen. $x_{m, n}^{\text {alt }}$ bezeichnet dabei die Anteile des zum Entscheidungszeitpunkt bereits vorhandenen Portfolios.

Der Preis $p_{m, n}$ eines Services richtet sich insbesondere nach dem zugrunde liegenden Lizenzmodell. Im Wesentlichen werden hier fünf Lizenzmodelle diskutiert (vgl. bspw. Boles und Schmees 2003, S. 388ff). Zeitlizenzen, bei denen der Nachfrager beliebig oft Services auf eine definierte Weise nutzen kann, werden im Modell abgebildet, indem der Preis für die einmalige Durchführung eines Service null gesetzt wird und die Lizenzkosten als Fixkosten für die Einbindung (s. u.) berücksichtigt wird. Bei Mengenlizenzen bezahlt der Kunde für die Anzahl der Nutzung eines oder mehrerer Services. Ist diese Anzahl gleich eins, so spricht man von „pay-per-use“. Für Mengenlizenzen ist das Modell - wie beschrieben - anwendbar, indem der Preis pro Ausführung berücksichtigt wird. Als eine Art Mischform aus Zeit- und Mengenlizenzen, lassen sich Intensitätslizenzen, bei denen nach Anzahl der Nutzungen pro Zeiteinheit abgerechnet wird, ebenfalls abbilden. Für die Berücksichtigung von Parameterund Ressourcenlizenzen, bei welchen sich der Preis anhand der Aufrufparameter bzw. Ressourcenausnutzung errechnet, sind jeweils die Werte aus den - auch herkömmlich zur Entscheidung über die Einbindung eines Service erstellten - Vorkalkulationen heranzuziehen. 
Aus diesen Vorkalkulationen gilt es die durchschnittlichen Preise für eine Serviceausführung als Planwert im Modell zu berücksichtigen. Nichtsdestotrotz kann es natürlich im Einzelnen auch komplexere Preismodelle geben, die nicht ohne Weiteres abgebildet werden können. Dennoch bietet die hier vorgeschlagene Modellierung mit mehreren Inputparametern Möglichkeiten wichtige Preismodelle zu berücksichtigen.

Die Kosten $C_{a_{\bullet, n}}$ je Lieferant $n$ ergeben sich mit (3.1) als Produkt aus Einbindungskostensatz $c_{a_{\bullet, n}}$ und der Differenz aus der Anzahl der Einbindungen von Services des Lieferanten $n$ im neuen Portfolio und der Einbindungen im alten Portfolio. Daneben entspricht die Anzahl aller Einbindungen wiederum der Summe der im alten und im neuen Portfolio eingebundenen Services (mit Hilfe der Signum-Funktion, welche für positive Werte den Funktionswert 1, für negative den Funktionswert -1 und für 0 den Funktionswert 0 liefert, wird abgeprüft, ob für einen Service $a_{m, n}$ der Portfolioanteil $x_{m, n}>0$ oder $x_{m, n}=0$ ist und somit letztlich, ob Einbindungskosten $c_{a_{\mathbf{0}, n}}$ anfallen oder nicht). Dabei werden Erfahrungskurveneffekte jeweils mittels des Exponenten $\gamma$ berücksichtigt.

$$
\text { (3.1) } C_{a_{\mathbf{v}, n}}=c_{a_{\mathbf{v}, n}} \cdot\left[\left(\left(\sum_{m=1}^{M}\left(\operatorname{sgn}\left[x_{m, n}\right]-\operatorname{sgn}\left[x_{m, n}^{a l t}\right]\right) \cdot \operatorname{sgn}\left[x_{m, n}\right]\right)+\sum_{m=1}^{M} \operatorname{sgn}\left[x_{m, n}^{\text {alt }}\right]\right)^{\gamma}-\left(\sum_{m=1}^{M} \operatorname{sgn}\left[x_{m, n}^{\text {alt }}\right]\right)^{\gamma}\right] .
$$

Da in der Realität meist Liefer- bzw. Kapazitätsbeschränkungen für Services existieren, können für einen Service $a_{m, n}$ Lieferbeschränkungen im Sinne einer minimal bzw. einer maximal angebotenen Liefermenge (d. h. die minimal und maximal möglichen Serviceausführungen durch einen Dienstleister) existieren. Diese sind in Nebenbedingungen der Form $Q x \leq q$ abzubilden (System linearer Ungleichungen). Dieses Ungleichungssystem besitzt eine Zeile für jede Lieferrestriktion und $M \cdot N$-Spalten.

Ebenfalls mit Nebenbedingungen können auch Mengenrabatte oder Preisstaffeln abgebildet werden, so dass kein Festpreis angenommen werden muss. Zudem könnte die vereinfachende Annahme des einheitlichen Kostensatzes $c_{a_{\mathbf{v}, n}}$ ohne Weiteres aufgehoben und individuelle Kostensätze definiert werden, die dann in einem Vektor $c_{a_{\bullet}, n}$ enthalten sind. Die Vektoren $c_{a_{\mathbf{o}, n}}$ eines jeden Lieferanten $n$ wären dann später mit der Matrix $A$ zu multiplizieren.

(A.3) Für die erstmalige Einbindung eines Lieferanten $n$ fallen die Kosten $C_{\bullet, n}$ (diese enthalten auch Koordinations- und Transaktionskosten) an.

Damit ergeben sich die gesamten Einbindungskosten eines Portfolio $C_{P}$ wie folgt aus der Summe der Einbindungskosten für jeden neuen Lieferanten und der Einbindungskosten für alle neuen Services $C_{a_{\bullet, n}}$ :

$$
C_{P}=\sum_{n=1}^{N}\left(C_{\bullet, n} \cdot\left(1-\operatorname{sgn}\left[\left(1-\operatorname{sgn}\left[\sum_{m=1}^{M} x_{m, n}\right]\right)+\operatorname{sgn}\left[\sum_{m=1}^{M} x_{m, n}^{a l t}\right]\right]\right)+C_{a_{\bullet, n}}\right) .
$$


Mit Hilfe der Signum-Funktionen wird abgeprüft, ob die Einbindungskosten $C_{\bullet, n}$ für den Lieferanten $n$ anfallen oder nicht, denn aus dem Klammerausdruck $\left(1-\operatorname{sgn}\left[\left(1-\operatorname{sgn}\left[\sum_{m=1}^{M} x_{m, n}\right]\right)+\operatorname{sgn}\left[\sum_{m=1}^{M} x_{m, n}^{\text {alt }}\right]\right]\right)$ ergibt sich nur dann 1, wenn Services dieses Lieferanten im neu umzusetzenden Portfolio enthalten sind, im alten jedoch nicht. In allen anderen Fällen wird dieser Ausdruck 0.

Zudem ist davon auszugehen, dass Lieferanten ihre Services nicht mit Sicherheit pünktlich oder in der vereinbarten Qualität liefern. Für diese Unsicherheit muss eine erwartete Wahrscheinlichkeit als Zufallsvariable gebildet werden, die realistischerweise - da Services sowohl in größerem bzw. kleinerem Umfang als ex ante geschätzt ausfallen können - streuen kann. Insofern soll gelten:

(A.4) Die Wahrscheinlichkeit $w_{m, n}$ dafür, dass der Service $a_{m, n}$ eines Lieferanten $n$ für Aktivität $m$ nicht in vereinbarter Lieferqualität erbracht wird, ist durch normalverteilte Zufallsvariablen abgebildet, deren Erwartungswerte $E\left(w_{m, n}\right)$ ist. Als Streuungsmaß wird die Standardabweichung $\sqrt{\operatorname{Var}\left(\mathcal{W}_{m, n}\right)}$ verwendet.

Für die Wahrscheinlichkeit $w_{m, n} \in[0 ; 1]$ bedeutet $w_{m, n}=0$, dass der Service $a_{m, n}$ mit Sicherheit geliefert wird bzw. $w_{m, n}=1$, dass er in jedem Fall nicht geliefert wird.

(A.5) Tritt der Fall ein, dass Service $a_{m, n}$ nicht in vereinbarter Qualität geliefert wird, fallen zusätzliche Kosten (bspw. durch Umdisponierung auf einen anderen Service, Schäden durch temporären Prozesssausfall abzgl. etwaig vereinbarter Konventionalstrafen) in Höhe von $k_{m, n}$ mit $k_{m, n} \geq 0$ an.

Wegen (A.2), (A.4) und (A.5) ist die Höhe der erwarteten Auszahlungen $\mu_{m, n}$ für eine Einheit des Services $a_{m, n}$ wie folgt:

$$
\mu_{m, n}=p_{m, n}+E\left(w_{m, n}\right) \cdot k_{m, n}
$$

Da die Wahrscheinlichkeiten $w_{m, n}$ normalverteilt sind, gilt dies für die Auszahlungen ebenso. Die zugehörige Standardabweichung $\sigma_{m, n}$ der erwarteten Auszahlungen $\mu_{m, n}$ einer Einheit $a_{m, n}$ ergibt sich mit

$$
\sigma_{m, n}=\sqrt{\operatorname{Var}\left(w_{m, n}\right)} \cdot k_{m, n}
$$

Die Wahrscheinlichkeit für einen Ausfall ist selbst bei konkurrierenden Services zweier Lieferanten nicht unabhängig. Hier können Ereignisse existieren, wie bspw. die NichtVerfügbarkeit einer Internet-Datenbasis, auf die beide zugreifen müssen, die zum Ausfall beider Services führen. Deshalb wird angenommen:

(A.6) Zwischen den Ausfallwahrscheinlichkeiten $w_{m, n}$ zweier Services unterschiedlicher Lieferanten $n_{i}$ und $n_{j}$ mit $n_{i} \neq n_{j}$, die für gleiche oder verschiedene Aktivitäten $m_{i}$ und $m_{j}$ eingesetzt werden können, bestehen lineare Abhängigkeiten. Diese sind über die 
Korrelationskoeffizienten $\rho\left(a_{m_{i}, n_{i}}, a_{m_{j}, n_{j}}\right)$ abgebildet und werden als horizontale Korrelationen bezeichnet.

Betreibt ein Anbieter von Services bspw. eine Infrastruktur für mehrere Services, so könnte deren Lieferqualität durch den Ausfall der Infrastruktur in Folge von Wartungsmaßnahmen, Hardwareschäden etc. beeinträchtigt werden. Deshalb wird folgende Annahme getroffen:

(A.7) Zwischen den Ausfallwahrscheinlichkeiten $w_{m, n}$ zweier Services des gleichen Lieferanten existieren lineare Abhängigkeiten, die über die Korrelationskoeffizienten $\rho\left(a_{m_{i}, n}, a_{m_{j}, n}\right)$ abgebildet werden sollen (hier als vertikale Korrelation bezeichnet).

Auf Basis dieser Annahmen kann ein optimales ITSP zur Durchführung aller betrachteten Prozesse zusammengestellt werden. Hierzu sind die optimalen Anteile $x_{m, n}^{*}$ zu ermitteln. Die erwarteten Auszahlungen $\mu_{p}$ für das ITSP errechnen sich als Summe der gewichteten erwarteten Auszahlungen aller im ITSP berücksichtigten Services.

$$
\mu_{P}=\sum_{m=1}^{M} \sum_{n=1}^{N} \mu_{m, n} \cdot x_{m, n}
$$

Um das optimale ITSP zu ermitteln, ist die Risikoeinstellung des Entscheiders zu berücksichtigen, d. h. es ist eine Abwägung zu treffen wie viel Risiko (Gefahr von Service-Ausfällen wächst) er bereit ist einzugehen, um die Auszahlungen für Services zu verringern. Gemäß dem Bernoulli-Prinzip wird das Entscheidungsverhalten aufgrund des erwarteten Nutzens bestimmt, der allen möglichen ITSP zugeordnet wird. Dieser Nutzen lässt sich mit Hilfe von Risikopräferenzfunktionen ermitteln. Hierzu wird folgende Annahme getroffen:

(A.8) Für den Entscheider existiert eine Nutzenfunktion, die mit dem Bernoulli-Prinzip vereinbar ist und jedem ITSP einen Nutzen zuordnet. Der Nutzen eines Portfolios ist gleich dem erwarteten Nutzen dieser Alternative. Der Entscheider wählt stets (wegen der zu Grunde liegenden Auszahlungsperspektive) dasjenige ITSP, welches den minimalen Präferenzfunktionswert aufweist.

Das Portfoliorisiko ist - ebenso wie bei den einzelnen Services - als Wahrscheinlichkeit negativer und positiver Abweichung von den erwarteten Auszahlungen des Portfolios zu verstehen. Es wird daher angenommen:

(A.9) Das Risikomaß ist die Kovarianz der erwarteten Auszahlungen für den einzelnen Service $a_{m, n}$ zu den erwarteten Auszahlungen des ITSP.

Das Risiko ${\sigma_{P}}^{2}$ des Portfolios entspricht damit der Summe aller Kovarianzen, die mit den Anteilen $x_{m, n}$ der IT-Services gewichtet wurden:

$$
\sigma_{P}^{2}=\sum_{m_{i}=1}^{M} \sum_{n_{i}=1}^{N} \sum_{m_{j}=1}^{M} \sum_{n_{j}=1}^{N} x_{m_{i}, n_{i}} x_{m_{j}, n_{j}} \operatorname{COV}\left(a_{m_{i}, n_{i}}, a_{m_{j}, n_{j}}\right)
$$

Die Kovarianzen lassen sich mit Hilfe von

$$
\operatorname{COV}\left(a_{m_{i}, n_{i}}, a_{m_{j}, n_{j}}\right)=\sigma_{m_{i}, n_{i}} \cdot \sigma_{m_{j}, n_{j}} \cdot \rho\left(a_{m_{i}, n_{i}}, a_{m_{j}, n_{j}}\right)
$$


bestimmen.

Die Varianzen $\sigma_{m, n}^{2}$ entsprechen jeweils den Einzelrisiken der Services, d. h. der Verletzung der Lieferqualität, die mit einer Wahrscheinlichkeit $w_{m, n}$ eintritt. Dagegen lässt sich die Summe aller übrigen Kovarianzen als Maß für die Höhe des Verbundrisikos interpretieren, welches im Portfolio enthalten ist.

Alle Portfolios mit minimalem Risiko zu je einer gegebenen erwarteten Auszahlung liegen auf einer Kurve. Je niedriger die Wahrscheinlichkeiten für die Nichteinhaltung der Lieferqualität $w_{m, n}$ und damit die Auszahlungsrisiken der einzelnen Leistungen korreliert sind, desto höher sind die erzielbaren Diversifikationsvorteile. Entsprechend sind sie maximal, wenn die Risiken der Leistungen perfekt negativ miteinander korreliert sind. Die Kurve der Kombinationen mit minimalem Risiko ist immer stetig und in $d \geq 1$ Kurvenabschnitten abschnittsweise definiert. Die Auszahlungshöhe und das zugehörige Risiko ergeben sich für jeden Punkt eines Kurvenabschnitts aus der Linearkombination $\left(x_{d}^{u}+\lambda_{2}^{d} w_{d}^{o}\right)$ zweier Gewichtungsvektoren des Kurvenabschnitts (Corner Portfolios), wobei für $\lambda_{2}^{d}$ jeweils nur ein kurvenabschnittspezifisches Intervall zulässig ist. Aus der Menge aller Portfolios sind dabei nur diejenigen effizient, die bei gegebenen erwarteten Auszahlungen die minimale Varianz bzw. bei gegebener Varianz die minimalen erwarteten Auszahlungen aufweisen.

Der Entscheider wählt dasjenige ITSP, das seiner Risikoeinstellung entspricht. Schneeweiß hat gezeigt, dass im Falle einer Konstellation, wie sie durch die Annahmen (A.3), (A.4) und (A.8) gegeben ist, nur ein Präferenzfunktional der folgenden Form mit dem Bernoulli-Prinzip vereinbar ist (Schneeweiß 1967, S. 149):

$$
\varphi^{\prime}\left(\mu_{P}, \sigma_{P}, C_{P}\right)=\mu_{P}+\frac{b}{2} \cdot \sigma_{P}^{2}+\frac{C_{P}}{\sum_{i=1}^{M} i_{M}} \rightarrow \min
$$

Der Präferenzparameter $b$, der beispielsweise mittels des Arrow-Pratt Maßes ermittelt werden kann, bildet dabei die Risikoeinstellung (Schneeweiß 1967, S. 90) des Entscheiders ab, die für $b>0$ Risikoaversion, für $b=0$ Risikoneutralität und für $b<0$ Risikofreude widerspiegelt.

Da die in der Zielfunktion (3.8) enthaltene Kostenfunktion $C_{P}$ wegen der Sprungstelle, die sich aufgrund der Fixkosten ergibt, weder stetig noch differenzierbar ist, kann der allgemeine Markowitz-Algorithmus nicht mehr Verwendung finden. Kellerer et al. (2000) gehen auf diese Problematik ein und zeigen, dass es sich hierbei um ein NP-hartes Problem handelt. Eine vollständige Enumeration aller möglichen Portfoliokombinationen zur Lösung des Problems ist hier kein realisierbarer Weg, da dies bei $M$ Aktivitäten und $N$ Lieferanten die Kalkulation von $\left(2^{N}-1\right)^{M}$ Portfolios bedeutet, falls die Angebotsmatrix voll besetzt ist. Deshalb wurden für die Ermittlung des optimalen ITSP bei Einbeziehung neuer Lieferanten und Services zwei Algorithmen (,, $A d d$ “- und ,Subtract“ Algorithmus) entwickelt, mittels derer sich jeweils eine approximative Lösung unter Berücksichtigung der Einbindungs- und Wechselkosten für das Optimierungsproblem ermitteln lässt. Da im Mittelpunkt des Beitrags jedoch die Idee des ITSP steht, wird hier nicht weiter darauf eingegangen. 


\section{Modellanwendung und Ergebnisinterpretation}

Nachstehend ist das Fallbeispiel eines FDL beschrieben, mit dem zusammen die Datenbasis für eine Modellanwendung erstellt wurde. Aus Anonymisierungsgründen wurden die Daten hierbei zwar verändert, ohne jedoch die Aussagen aus dem Modell zu modifizieren. Daneben wird auch auf die bei der Modellanwendung aufgetretenen kritischen Punkte eingegangen.

Der FDL ist als Multikanalbank im deutschen Markt positioniert. Zur Standardisierung und Verbesserung seiner Prozesse will er u. a. die eigene Anwendungslandschaft nach dem Vorbild SOA umbauen. Ein konkretes Ziel ist hierbei die Beratungs- und Verkaufsfunktionalitäten für alle Vertriebskanäle weitgehend einheitlich bereitzustellen. Dabei sollen einzelne Aktivitäten der Beratungs- und Verkaufsprozesse durch Services realisiert werden. Bild 2 zeigt den Prozess zur Girokontoeröffnung vereinfacht im Überblick.

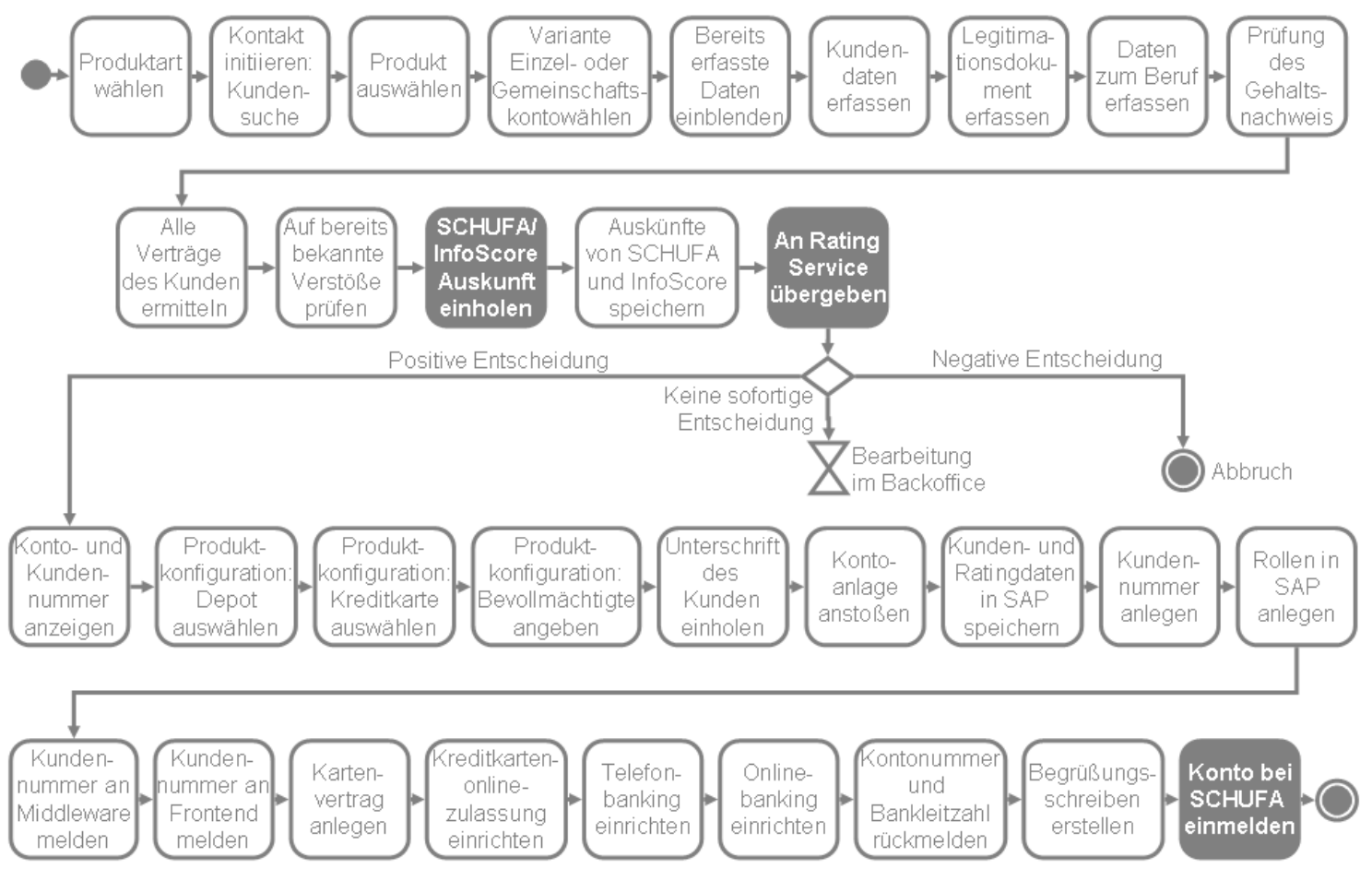

\section{Bild 2: Prozess zur Girokontoeröffnung}

Exemplarisch werden aus diesem Prozess die drei markierten Aktivitäten SCHUFA/InfoScore Auskunft einholen, An Rating Service übergeben und Konto bei SCHUFA einmelden herausgegriffen. Sie können jeweils von Services durchgeführt werden, die entweder von der eigenen IT-Tochter oder von zwei externen Anbietern (bspw. SCHUFA, die einen Zugriff mittels XML-Gateway anbietet) stammen. Hintergrund ist hier, dass das Girokonto als strategisches Einstiegsprodukt für Neukunden angesehen und deshalb stark beworben wird. Aufgrund der dadurch erwarteten höheren Anzahl an Kontoeröffnungen war im Vorfeld die Entscheidung über die Einbindung der Services zu treffen. Die Tabelle 3 zeigt die Datenbasis.

Die Preise für die Services können dabei noch relativ einfach anhand von Angeboten der Anbieter bzw. von Verrechnungspreisen der IT-Tochter ermittelt werden. Gleiches gilt für die Zuverlässigkeit und damit für die erwartete Ausfallwahrscheinlichkeit. Hier sicherten die An- 
bieter in den angeforderten Service Level Agreements (SLA) eine mittlere Verfügbarkeit zu (ggf. mit Schwankungsbreite), die interessanterweise - wohl auch wegen der z. T. neuen Technologie - durchaus unterschiedlich waren. So liegt bei Lieferant B die zugesicherte Verfügbarkeit des Service für Auskunft einholen bei über 99\%, wohingegen sie bei der SCHUFA Einmeldung unter 90\% liegt. Daneben konnten auch die Kosten bei Ausfall eines Service noch relativ gut geschätzt werden, da hier v. a. diejenigen Kosten anzusetzen sind, die entstehen, wenn durch den Service-Ausfall eine Verzögerung im Giroeröffnungsprozess auftritt. Da bspw. Verzögerungen bei der Einmeldung des Kontos für den Kunden nicht erkennbar werden - der Prozessschritt erfolgt erst nach Begrüßung des Kunden - sind die Umdisponierungskosten hier eher gering. Schwierig wurde es bei der Ermittlung der Einbindungskosten. Dies konnte nur auf Basis von Erfahrungswerten eines am Projekt beteiligten Beratungsunternehmens erfolgen, das hierfür aus anderen Projekten Werte bereitstellte. Die Einbindungskosten für die Services der eigenen IT-Tochter wurden dabei in Rücksprache mit dieser als viel geringer angegeben. Hier wurden Einbindungskosten in Höhe von $C_{\bullet, 1}=1.000$ $\epsilon$, bei Lieferant A $C_{\bullet, 2}=5.500 €$ und bei Lieferant $\mathrm{B} C_{\bullet, 3}=5.250 €$ zugrunde gelegt. Zudem wurde in der betrachteten Periode mit 15.000 Durchläufen des Giroeröffnungsprozesses gerechnet. Davon kommt es in 9.000 Durchläufen auch zum Vertragsabschluss - so die Schätzung aufgrund der Erfahrungen in den letzten Perioden und von Prognosen seitens des Privatkundenbereichs.

Tabelle 3: Daten verfügbarer Services

\begin{tabular}{|c|c|c|c|}
\hline & Eigene IT-Tochter & Lieferant A & Lieferant B \\
\hline \multicolumn{4}{|c|}{ Aktivität 1: SCHUFAInfoScore Auskunft einholen $\left(i_{l}=15.000\right)$} \\
\hline$p_{l, n}$ & 1,30 & 1,20 & 1,10 \\
\hline$E\left(w_{l, n}\right)$ & 0,075 & 0,050 & 0,025 \\
\hline $\operatorname{Var}\left(w_{l, n}\right)$ & 0,001 & 0,003 & 0,004 \\
\hline$k_{l, n}$ & 6,00 & 6,00 & 6,00 \\
\hline$C_{1_{\bullet, n}}$ & 10.000 & 11.000 & 10.500 \\
\hline \multicolumn{4}{|c|}{ Aktivität 2: An Rating Service übergeben $\left(i_{2}=15.000\right)$} \\
\hline$p_{2, n}$ & 1,50 & 1,60 & 1,70 \\
\hline$E\left(w_{2, n}\right)$ & 0,023 & 0,066 & 0,108 \\
\hline $\operatorname{Var}\left(w_{2, n}\right)$ & 0,009 & 0,005 & 0,003 \\
\hline$k_{2, n}$ & 5,30 & 5,30 & 5,30 \\
\hline$C_{2 \cdot, n}$ & 8.000 & 8.800 & 8.400 \\
\hline \multicolumn{4}{|c|}{ Aktivität 3: Konto bei SCHUFA einmelden $\left(i_{3}=9.000\right)$} \\
\hline$p_{3, n}$ & 2,25 & 1,84 & 2,70 \\
\hline$E\left(w_{3, n}\right)$ & 0,125 & 0,133 & 0,083 \\
\hline $\operatorname{Var}\left(w_{3, n}\right)$ & 0,16 & 0,2567 & 0,1296 \\
\hline$k_{3, n}$ & 1,20 & 1,20 & 1,20 \\
\hline$C_{3 \cdot n}$ & 14.000 & 15.400 & 14.700 \\
\hline
\end{tabular}

Auf Basis dieser zusammen mit dem FDL ermittelten Datenbasis, wurde nunmehr das in Kapitel 3 beschriebene Modell angewendet. Zur Veranschaulichung wurden für einen risikoneut- 
ralen, einen schwach respektive stark risikoaversen Entscheider die Ergebnisse im Vergleich (Tabelle 4) dargestellt. Der risikoneutrale Entscheider (und dies wäre auch die Entscheidung des FDL bisher gewesen) würde für jede Aktivität ausschließlich den günstigsten Service einbinden und damit an jeden Anbieter einen Service vergeben (gekennzeichnet in der Tabelle durch den Anteil von eins). Je stärker jedoch der Entscheider auf das Risiko achtet, desto stärker wird das Volumen auf die drei Anbieter verteilt. Während das Service-Portfolio bei schwacher Risikoaversion pro Aktivität schon auf zwei Anbieter verteilt ist, sind es bei starker Risikoaversion sogar alle drei. Daran ist deutlich zu erkennen, dass ein Hedging des Ausfallrisikos mit steigender Risikoaversion bedeutender wird. Auffällig am Ergebnis ist auch, dass die vom risikoneutralen Entscheider gewählten Services mit dem niedrigsten Preis bei gleichzeitig höherem Ausfallrisiko mit steigender Risikoaversion nur noch einen geringen Anteil am Portfolio einnehmen. So wird bei schwacher Risikoaversion für jede Aktivität der günstigste Service nur noch zu einem Drittel gegenüber einem anderen Service zu zwei Drittel gewählt.

\section{Tabelle 4: Ergebnistableau im Fallbeispiel}

\begin{tabular}{|c|c|c|c|c|}
\hline \multicolumn{2}{|c|}{ Entscheidungssituation } & risikoneutral & schwach risikoavers $(b=5)$ & stark risikoavers $(b=40)$ \\
\hline \multirow{8}{*}{ 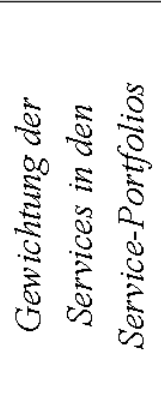 } & $x_{1,1}$ & 0 & 0 & 0,60 \\
\hline & $x_{1,2}$ & 0 & 0,64 & 0,22 \\
\hline & $x_{1,3}$ & 1 & 0,36 & 0,18 \\
\hline & $x_{2,1}$ & 1 & 0,31 & 0,16 \\
\hline & $x_{2,2}$ & 0 & 0,69 & 0,25 \\
\hline & $x_{2,3}$ & 0 & 0 & 0,59 \\
\hline & $x_{3,1}$ & 0 & 0,65 & 0,38 \\
\hline & $x_{3,2}$ & 1 & 0,35 & 0,14 \\
\hline & $x_{3,3}$ & 0 & 0 & 0,48 \\
\hline
\end{tabular}

Eine Aufteilung auf mehrere Services mehrerer Anbieter ist also bei Risikoaversion nicht nur vorteilhaft. Hier zeigt sich, dass eine Berücksichtigung des Ausfallrisikos im ITSP und damit die Service-Portfoliobildung umso wichtiger sind, je höher die zugrunde gelegte Ausprägung des Risikoaversionsparameters ist. Vielmehr wurde für den FDL transparent, dass die im Zuge der Umstellung auf eine SOA geplante Konsolidierung der Service-Lieferanten und damit einhergehende Konzentration auf einen Lieferanten (neben der eigenen IT-Tochter), nicht wie erhofft uneingeschränkt positiv zu beurteilen ist: Dem Effekt der Kosteneinsparung steht eine erhöhte Risikoposition entgegen, die jenen sogar mehr als aufwiegt. Gerade beim wichtigen Prozess der Giroeröffnung trug diese Erkenntnis zur Entscheidung bei, je Aktivität - trotz der höheren Kosten - zwei Lieferanten (schwach risikoaverse Lösung) einzubinden.

\section{Implikationen, kritische Würdigung und Ausblick}

Um den Einfluss von IOS auf die Abhängigkeiten von Lieferanten zu analysieren, wurde im vorliegenden Beitrag ein Modell entwickelt, mit dem sich ITSP unter Berücksichtigung von Kosten- und Risikogrößen optimieren lassen. Neben der Anwendung des Ansatzes mittels einer Datenbasis bei einem FDL werden im Weiteren die Modellzusammenhänge - um all- 
gemeine Aussagen zu erhalten - untersucht, um den zukünftigen Einfluss von IOS auf die in Bild 1 dargestellten Abhängigkeiten zu ermitteln.

\section{Schneller, flexibler Austausch bei Service-Ausfall durch semantische Konzepte}

Dem Umstand, dass Services realistischerweise hinsichtlich benötigter Menge, Zeitpunkt und Qualität nicht verfügbar sein können, wird im Modell in zweierlei Weise Rechnung getragen. Zum einen können Ausfallwahrscheinlichkeiten für einzelne Services berücksichtigt werden. Andererseits werden durch Beschreibung horizontaler und vertikaler Korrelationen Abhängigkeiten der Ausfälle von Services einbezogen. Durch letztere lassen sich „Klumpenrisiken“ abbilden, die sich bspw. darauf begründen, dass ein Prozess mittels Services eines Lieferanten bzw. vernetzter Lieferanten durchgeführt wird. Fällt demnach ein Service aus und kann - wie in der heutigen Praxis üblich - kein flexibler Austausch erfolgen, drückt sich das in hohen Umdisponierungskosten aus. Diese umfassen den Wechsel auf einen anderen Service und den ökonomischen Schaden, der durch die Verzögerung des Prozessdurchlaufs entsteht.

Setzt sich zukünftig eine teilautomatisierte Umdisponierung bei Service-Ausfall auf Basis einer semantischen Auszeichnung, Ontologie-Standards sowie Semantic-Web-Service Kompositionsansätze durch - so wie prototypisch heute in Ansätzen bereits möglich -, lassen sich die Umdisponierungskosten reduzieren. Je stärker sich diese reduzieren, desto geringer wird auch das Risiko des Service-Ausfalls im Portfolio bewertet (vgl. (3.1) und (3.2)). Falls die Umdisponierungskosten im Extremfall sogar gegen null gehen würden, entsteht bei einem Service-Ausfall kein ökonomischer Schaden mehr, d. h. es müsste ex ante kein Portfolio gebildet werden. Es würde genügen, jeweils den für eine Aktivität günstigsten Service anzufordern (Auszahlungsminimierung ohne Betrachtung des Risikos) und diesen bei Ausfall durch den zweitgünstigsten etc. zu ersetzen. Insofern widerlegen die Ergebnisse des Modells das zweite Prinzip von Kleindorfer und Saad (2005), welches besagt, dass Portfoliobildung immer die dominante Strategie ist.

Dies weist jedoch auch auf einen anderen Zusammenhang hin: Sollten sich die Umdisponierungskosten zukünftig stark vermindern lassen, dann könnten sich Unternehmen auch in zunehmendem Maße die Kosten für die Risikobewertung verfügbarer Services sparen. Es würde die Anbindung an ein Diensteverzeichnis genügen, das den jeweiligen Service-Preis aufführt.

\section{Schnelle Umsetzung geänderter Anforderungen und Reduzierung der Wechselkosten durch Service-Auszeichnung}

Beim Wechsel eines Lieferanten sind heute hohe Kosten und lange Umstellungszeiten in Kauf zu nehmen. Dies erhöht die Abhängigkeit von Lieferanten. Das Modell berücksichtigt diesen Effekt, indem beim Service-Wechsel die Kosten für die Einbindung eines bisher noch nicht genutzten Service (Kosten $C_{a_{\mathbf{b}, n}}$ ) einbezogen werden können. Auch die in der Praxis entstehenden hohen Kosten bei Wechsel auf einen neuen Dienstleister lassen sich im Modell mittels der Einbindungskosten $C_{\bullet, n}$ berücksichtigen. 
Sollten sich mit Hilfe semantischer Service-Auszeichnungen, Servicekomposition und SOA die Einbindungskosten eines Service bzw. eines neuen Lieferanten verringern lassen, wie würde sich dies auswirken? Lässt man sie im Extremfall gegen null gehen, so gewinnt das zweite Prinzip von Kleindorfer und Saad (2005) stark an Bedeutung, welches besagt, dass Portfoliobildung das Risiko reduziert. Der Vorteil beruht hier auf den berücksichtigten Abhängigkeiten bei Service-Ausfällen: Eine Risikoreduktion lässt sich allgemein immer dann erzielen, wenn zwei Services nicht perfekt miteinander korreliert sind. Je geringer die Korrelationen sind, desto größer sind die erzielbaren Diversifikationsvorteile und damit die Tendenz zu Portfolios mit vielen Lieferanten. Dieser Entwicklung können hohe Einbindungskosten gegenüber stehen (die auch die höheren Koordinations- und Transaktionskosten bei einer Vielzahl von Lieferanten enthalten), d. h. übersteigen diese die Diversifikationsvorteile, so besteht das ITSP tendenziell aus wenigen Services. Können sich die vorgenannten IOS jedoch durchsetzen, so wird dies gerade die (manuellen) Service-Einbindungskosten reduzieren. In der Tendenz würde dann die gesamte Durchlaufmenge einer Aktivität auf viele Services verteilt werden, um Diversifikationsvorteile zu nutzen. Eine Risikobetrachtung gewinnt demnach - im Vergleich zum letzten Punkt - an Bedeutung.

\section{Entscheidung über die Auslagerung einzelner Prozessaktivitäten}

Auch die Möglichkeit, die Auslagerung von Prozessen granularer zu gestalten, ist zu analysieren.

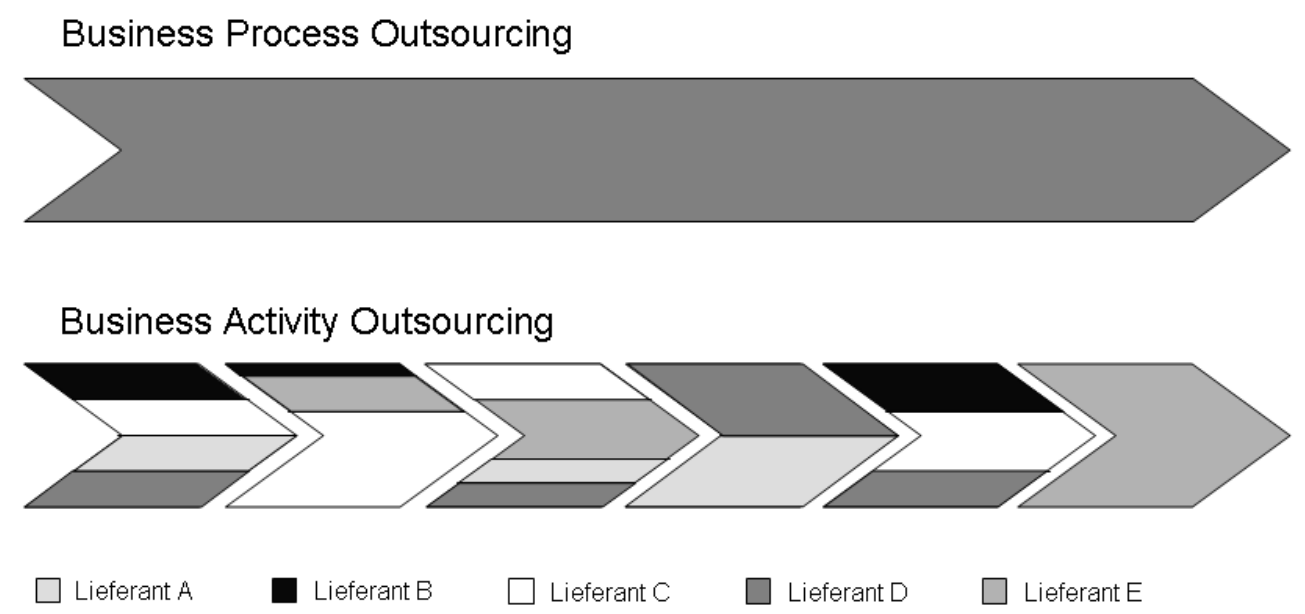

Bild 3: Vom BPO zum Business Activity Outsourcing

Die Ergebnisse verdeutlichen, dass mit der heute oftmals üblichen Praxis, einen Prozess vollständig - sowohl in den einzelnen Aktivitäten, als auch in der Durchlaufmenge einer Aktivität - an einen Dienstleister zu vergeben, ggf. Optimierungspotenzial verschenkt wird (vgl. Bild 3): Durch die Verteilung auf mehrere Dienstleister, ist bei einer nicht perfekten Korrelation das Portfoliorisiko geringer als die Summe der „Einzelrisiken“ der Services. Portfoliobildung lässt sich ausgehend vom BPO in zwei Richtungen vorantreiben: Mittels Unterteilung in Prozessaktivitäten, die dann an jeweils mehrere Dienstleister vergeben werden können, und mittels mengenmäßiger Verteilung einer Aktivität auf mehrere Services. 
Bisher war Business Activity Outsourcing jedoch oftmals eine unrentable Maßnahme, da die Einbindungskosten der einzelnen Services i. d. R. den Diversifikationsvorteil deutlich dominieren. Aber auch hier gilt: Sollten sich durch IOS die Einbindungskosten verringern, so werden nachfragende Unternehmen diesen Vorteil künftig stärker abschöpfen können. Dienstleister dürften zunehmend für einzelne Aktivitäten eingebunden werden, was zu einer stärkeren Spezialisierung (Service-Markt) - aber damit auch zu Chancen für kleine Dienstleister - führen würde.

Mit IOS könnte zwar die Einbindung mehrerer Dienstleister effizient möglich sein. Ob ein Unternehmen tatsächlich mit vielen tausenden Lieferanten interagieren will, oder ob sich ähnlich wie in der Automobilindustrie Tier-1 Lieferanten herausbilden, die das Management weiterer, Tier-2 und Tier-3 Lieferanten übernehmen, bleibt abzuwarten. Hier könnte man zukünftig bspw. einzelne Aktivitäten selektiv zusammenfassen, die dann von einem Tier-1 Lieferanten insgesamt übernommen werden, um im Modell zu analysieren, ob Lieferanten grob oder fein granular integriert werden sollten.

Daneben ist auch zu untersuchen, ob es sinnvoll ist, die Durchlaufmenge einer Aktivität auf mehrere Dienstleister aufzuteilen, wobei natürlich auch hier die höheren Einbindungskosten zu berücksichtigen sind. Der Vorteil einer Aufteilung lässt sich an den Abhängigkeiten bei Service-Ausfällen veranschaulichen: Dass sich das Risiko immer dann reduzieren lässt, wenn zwei oder mehr Services nicht perfekt miteinander korreliert sind, lässt sich bei der Mengenaufteilung besonders nutzen. Je geringer die (horizontale) Korrelation ist, desto größer sind die erzielbaren Diversifikationsvorteile. Letztere können auch dazu führen, dass teure oder bezüglich des Preisrisikos schlechte Services trotzdem in einem optimalen Portfolio eingehen.

Die Dienstleister selbst werden durch die beschriebenen Effekte tendenziell austauschbarer und müssen sich überlegen, wie sie sich gegenüber Konkurrenten abgrenzen können. Eine reine Konzentration auf die Zuverlässigkeit ihrer Services scheint hier zweifelhaft. IOS haben zudem das Potenzial, die eingangs beschriebene Abhängigkeit von Unternehmen (insbesondere bei wenigen Abnehmern) von ihren Dienstleistern umzukehren, so dass - ähnlich wie in der Automobilindustrie aufgrund der Marktmacht zu beobachten - die Dienstleister immer stärker von den Unternehmen abhängig werden.

Ein Kritikpunkt am beschriebenen Modell ist - wie auch im Fallbeispiel deutlich wurde - die Erhebung der Inputparameter: Neben Einbindungskosten müssen für jeden Service Preis, sowie Erwartungswert und Standardabweichung der Lieferqualität ermittelt werden. Während sich der Preis verhältnismäßig leicht bestimmen lässt, besteht das Hauptproblem v. a. bei den Ausfallwahrscheinlichkeiten der Services und den Korrelationen. Sensitivitätsanalysen verdeutlichen im Beispiel, dass gerade die fixen Kostengrößen (bspw. Einbindungskosten für einen Lieferanten, Einbindungskosten für einen neuen Service) das ermittelte Ergebnis stabilisieren. Dies liegt daran, dass sich die Inputparameter der in einem ITSP enthaltenen Lieferanten und Services stärker „verschlechtern“ müssten, damit sich bspw. die Einbindung eines neuen Lieferanten (mit den damit einhergehenden fixen Einbindungskosten) trotzdem rechnet. Gerade weiche „Übergänge“ zwischen Portfolios, bei denen ein neuer Lieferant nur zum geringeren Teil im ITSP berücksichtigt wird, treten bei einer geringen Änderung der Parameter 
daher selten auf. Nichtsdestotrotz ist jedoch darauf hinzuweisen, dass sich das Modellergebnis bei einer größeren Änderung der Inputparameter (bspw. größere Schätzfehler) verändert (das Gegenteil wäre auch nicht sinnvoll), denn die Ergebnisse sind natürlich abhängig von den Parametern.

Die Bewertung oder das Rating von Lieferanten und insbesondere der Lieferzuverlässigkeit ist grundsätzlich kein neues Thema (vgl. auch Disselkamp und Schüller 2004; Dreyer 2000; Glantschnig 1994; Harting 1994; Janker 1994; Koppelmann 2000; Smytha und Clemens 1993; Wagner 2002), das durch die Bewertung von Services neu entsteht. Hier sind verschiedene Ansatzpunkte zu berücksichtigen:

1. Verfahren zur Bewertung des Lieferanten beziehungsweise dessen Lieferzuverlässigkeit: Bei diesen Verfahren erfolgt die Lieferantenbewertung mittels zurechenbarer Kriterien. Beispiele hierfür sind Liquiditätsanalysen beziehungsweise eine Analyse der Investitionstätigkeit des Dienstleisters. Dies zielt darauf ab, Ereignisse wie eine mögliche Insolvenz (Services würden ausfallen) oder eine verminderte Investitionstätigkeit (Einschränkung der Lieferfähigkeit) zu antizipieren. Ein anderes Verfahren ist das Total Cost Supplier Selection Model von Smytka und Clemens. Dieses beinhaltet neben Kostenkomponenten auch Risikofaktoren, welche die zukünftige Lieferqualität bewerten. Hier werden Erfahrungswerte bei der Termin-, Qualitäts- und Mengenzuverlässigkeit mit Prognosen über die zukünftige Leistungsfähigkeit aufgrund finanzieller Größen o. ä. integriert. Auf dieser Basis erfolgt eine Risiko-Klassifikation (bspw. in einer Risiko-Lieferfähigkeits-Matrix) der Dienstleister, wodurch Ausfallwahrscheinlichkeiten abschätzbar werden.

2. Erfahrungsbezogene vs. zukunftsbezogene Bewertung: Oben wurden Bewertungskriterien genannt, die aufgrund von Erfahrungswerten eine Interpolation für zukünftiges Verhalten versuchen. Dies bringt natürlich auch durchaus Probleme mit sich, nämlich insbesondere dann, wenn diese - aufgrund beispielsweise struktureller Änderungen in der Technologie des Dienstleisters - nicht mehr aussagekräftig für die zukünftige Zuverlässigkeit sind. Diesen Mangel versuchen zukunftsbezogene Bewertungen auszugleichen, indem sie die zukünftige finanzielle Basis des Dienstleisters, dessen Innovationskraft, Kooperationsfähigkeit oder Technologieabhängigkeit analysieren (vgl. Disselkamp und Schüller (2004)). Ein Technologiewechsel, der die zukünftige Lieferzuverlässigkeit steigert, kann so Berücksichtigung finden (bspw. werden die Werte in der Risiko-Lieferfähigkeits-Matrix erhöht).

3. (Teil-)Automatisierung und Bewertung der Schätzqualität: Insbesondere bei einer Vielzahl von Dienstleistern und Services ist eine (teil-)automatisierte Bewertung notwendig. Deshalb bieten einige Softwarehersteller (z. B. SAP, Peoplesoft oder Navison) entsprechende Werkzeuge mit unterschiedlichen Integrationsgraden (bspw. unter Einbeziehung externer Rating-Unternehmen und deren Daten). So kann mit der Software von Peoplesoft die Lieferzuverlässigkeit der Dienstleister kontinuierlich anhand von 80 Ratingkriterien ermittelt werden. Wichtig ist dabei natürlich, dass es - wie bei Prognosen üblich - nicht darum gehen kann, die Information über die Lieferzuverlässigkeit „mit Sicherheit“ ermit- 
teln zu wollen. Vielmehr geht es darum unter Einbeziehung verfügbarer Informationen eine unter Kosten-/Nutzenaspekten bestmögliche Schätzung abzugeben.

Die auf diese Weise geschätzten Daten könnten als nicht-funktionale Eigenschaften der ITServices in Dienstverzeichnissen (UDDI), die Daten zur Verfügbarkeit und Qualität enthalten, gespeichert werden. Die Menge der hier anfallenden Daten verdeutlicht jedoch, dass es durchaus sinnvoll ist, dieses Verzeichnis zentral zu betreiben (in diese Richtung sind wohl auch die Aktivitäten der oben genannten Softwarehersteller zu deuten). Lieferanten erhalten dadurch eine Plattform zum Angebot ihrer IT-Services mit allen funktionalen und nichtfunktionalen Eigenschaften. Um der Manipulationsgefahr bei einem Betrieb des Verzeichnisses durch einen Anbieter oder mehrere entgegenzutreten, wäre eine unabhängige dritte Instanz zum Betrieb besser geeignet. Hierdurch lässt sich sukzessive ein Bewertungssystem aufbauen, bei dem nachfragende Unternehmen bspw. auch Bewertungen der Zuverlässigkeit der Lieferung etc. einstellen können. Dagegen sind natürlich schwer kalkulierbare Einzelfälle wie Viren bzw. Internetwürmer, die zu weitreichenden Service-Ausfällen führen können, in einem Diensteverzeichnis kaum mit repräsentativen Schätzgrößen zu hinterlegen.

Die vorliegende Arbeit stellt einen Ansatz zur Optimierung eines ITSP anhand quantitativer Größen vor. Darüber hinaus konnten - unter der Voraussetzung der Etablierung von IOS eine Reihe allgemeiner Aussagen abgeleitet werden. So zeigt das Modell bei einer Optimierung von Auszahlungs- und Risikogrößen, dass sich durch die IOS die Abhängigkeit von externen Dienstleistern verringern wird, da Unternehmen dann zur Senkung des Ausfallrisikos mehrere Dienstleister einbinden werden. Dies lohnt sich, da die IOS eine Reduktion der Einbindungs- und Koordinationskosten sowie der Umdisponierungskosten versprechen. Anhand des Modells konnte weiterhin verdeutlicht werden, dass das Ausfallrisiko durch die Vergabe einzelner Aktivitäten an mehrere Dienstleister diversifiziert werden kann, d. h. die Ausführung einer Aktivität muss nicht immer durch den gleichen Dienstleister erfolgen. Durch diese Aufteilung lässt sich Optimierungspotenzial heben.

Weiterer Forschungsbedarf besteht einerseits in der Erweiterung des Ansatzes mit qualitativen Größen. Zum anderen soll auch die Seite der Anbieter betrachtet werden. Auch für diese stellt sich die Frage, wie sich IOS auf deren Marktposition und Service-Portfolio auswirken werden.

\section{Literatur}

Aalst, Wil M.P. van der (2003): Don't go with the flow: Web services composition standards exposed. In: IEEE Intelligent Systems 18 (1), S. 72-76.

Boles, Dietrich; Schmees, Markus (2003): Kostenpflichtige Web-Services, in: Uhr, Wolfgang; Esswein, Werner; Schoop, Eric (Hrsg.): Wirtschaftsinformatik 2003: Medien - Märkte - Mobilität, Tagungsband 6, Band I, S. 385 - 403.

Bruijn, Jos de; Fensel, Dieter; Keller, Uwe; Lara, Rubén (2005): Using the Web Service Modeling Ontology to Enable Semantic E-Business. In: Communications of the ACM 48 (12), S. 43-47.

Blodig, Christoph; Heinrich, Bernd; Wehrmann, Alexander (2006): Wirtschaftlichkeitsanalyse bei 
der Einführung und Gestaltung von Wissensmanagementsystemen am Beispiel der Kundenserviceprozesse einer Automobilbank. In: Lehner, F., Nösekabel, H., Kleinschmidt, P., (Hrsg.): Multikonferenz Wirtschaftsinformatik (MKWI) 2006, 2. Band, 2006, S. 473-486.

Buck, Konrad (2006): Salesforce will On-Demand-Module koppeln. In: Computer Zeitung 2006 (7), Seite 11.

Disselkamp, Marcus; Schüller, Rudolf (2004): Lieferantenrating - Instrumente, Kriterien, Checklisten, Gabler, Wiesbaden.

Dreyer, H.-W. (2000): Lieferantentypspezifische Bewertung von Lieferleistungen: eine empirische Analyse, Lang, Frankfurt/Main.

Glantschnig, E. (1994): Merkmalsgestützte Lieferantenbewertung, Fördergesellschaft ProduktMarketing, Köln.

Harting, D. (1994): Lieferanten-Wertanalyse - Ein Arbeitshandbuch mit Checklisten und Arbeitsblättern für Auswahl, Bewertung und Kontrolle von Zulieferern, 2. Auflage, Schäffer-Poeschel, Stuttgart.

Janker, C. G. (2004): Multivariate Lieferantenbewertung - Empirisch gestützte Konzeption eines anforderungsgerechten Bewertungssystems, Deutscher Universitäts-Verlag, Wiesbaden.

Kellerer, Hans; Mansini Renata; Speranza, Grazia (2000): Selecting Portfolios with Fixed Costs and Minimum Transaction Lots. In: Annals of Operations Research, 99 (1), 287-304.

Kleindorfer, Paul R.; Saad, Germaine H. (2005): Managing Disruption Risks in Supply Chains. In: Production and Operations Management 14 (1), S. 53-68.

Knolmayer, Gerhard (1991): Die Auslagerung von Servicefunktionen als Strategie des ISManagements. In: Heinrich, Lutz J.; Pomberger, Gustav; Schauer, Reinbert (Hrsg.): Die Informationswirtschaft im Unternehmen, Linz, S. 323-341.

Koppelmann, U. (2000): Beschaffungsmarketing. 3. Auflage, Springer Verlag, Berlin u. a.

Lara, Ruben; Roman, Dumitru; Polleres, Axel; Fensel, Dieter (2004): A conceptual Comparison of WMSO and OWL-S. In: Zhang, L.-J.; Jeckle, M. (Hrsg.): ECOWS 2004, LNCS 3250, Springer, Berlin, Heidelberg, S. 254-269.

Latour, Almar (2001): Trial by Fire: A Blaze in Albuquerque Sets Off Major Crisis for Cell-Phone Giants - Nokia Handles Supply Shock With Aplomb as Ercisson of Sweden Gets Burned - Was Sisu the Difference?“. In: Wall Street Journal vom 29.04.2001, S. A1.

Markowitz, Harry M. (1959): Portfolio Selection - Efficient Diversification of Investments. John Wiley, New York.

Martínez-de-Albéniz, Victor; Simchi-Levi, David (2005): A Portfolio Approach to Procurement Contracts. In: Production and Operations Mangement 14 (1), S. 90-114.

Meyer, Bertrand (1990): Objektorientierte Softwareentwicklung, Carl Hanser Verlag, München Wien.

o. V. (2005): Den Kurs korrigieren, CIO, 03.11.2005. http://www.cio.de/_misc/article/ print/index.cfm?pid=177\&pk=814497\&op=prn, Abruf am 12.06.2006.

Racky, Bardo (2005): Best Practices Outsourcing, CIO, Analystenkolumne, 21.09.2005. http://www.cio.de/_misc/article/print/index.cfm?pid=168\&pk=813272op= 
prn, Abruf am 12.6.2006.

Ran, Shuping (2003): A Model for Web Services Discovery With QoS. In: ACM SIGecom Exchanges 4 (1), S. 1-10.

Reinicke, Michael (2005): Eine ökonomische Bewertung der Dienstauswahlverfahren in serviceorientierten Overlaynetzen. In: Ferstl, O., Sinz, E., Eckert, S., Isselhorst, T., (Hrsg.): Wirtschaftsinformatik 2005 - eEconomy eGovernment eSociety, Bamberg, Heidelberg, S.129-148.

Schneeweiß, H. (1967): Entscheidungskriterien bei Risiko, Springer, Berlin.

Smytha, D.L.; Clemens, M.W. (1993): Total Cost Supplier Selection Model. In: International Journal of Purchasing and Material Management 1 (29), S. 42-49.

Takahashi, Sonoko; Davis, Euan; Parker, Andrew; King, Onica (2006): European Business Process Outsourcing Spending Forecast: 2006 To 2011. Forrester Research Trends, 26.09.2006.

Tomlin, Brian (2006): On the Value of Mitigation and Contingency Strategies for Managing Supply Chain Disruption Risks. In: Management Science 52 (5), S. 639-657.

Wagner, S. M. (2002): Lieferantenmanagement, Hanser, München.

Wirth, Andrea (1996): Technologische und institutionelle Aspekte bei der Bereitstellung von Informationsverarbeitungsleistungen. Europäische Hochschulschriften. Peter Lang, Bd. 1845. 